\title{
SUSTO: THE CONTEXT OF COMMUNITY MORBIDITY PATTERNS
}

Robert T. Trotter II Pan American University

Susto. the widespread Latin American folk illness, normally translated as fright or shock, holds a special place in medical anthropology. For the past 25 years or more it has provided a convenient springboard tor ethnographic descriptions, for explorations ot the effect of culture on health beliefs, and it has been a common analytical arena for the assessment of stress, social roles, sex roles, the naturenuture controversy, and the epistemology of medical anthropology itself. While this is a significant burden to load onto a single ailment, susto has withstood this analytical onslaught extremely well and still provides a useful focus for further investigation.

The earlier works on sustofail into three broad categories. First are rhose which provide a conventional ethnographic description of the illness (e.g., Rubel 1960 1966; Madsen 19(54; Nail and Speilberg 1967; Trotter and Chavira 1980, 1981). Beyond description, other authors have postulated the causes of susto from sociocultural perspectives (Gillen 1945; Rubel 1964; O'Neil and Seiby 1968; Uzzeil 19-4; O'Neil 1975; O'Neil and Rubel 19-6, 1980; Rubel and'O'Neil 19-9, and Logan 19^9), psychological and/or psychiatric perspectives (Gillen 1948; Sal y Rosas 1958; Kiev 1968; Goebel 19^5; Grebe and Segura 1974), and biological (Bolton 1980, 1981) perspectives. These two groups together attempt to answer the questions, "what is JV/J/Y??" "what is its cause, and its effect."" Collectively, these works constitute our current understanding ot the etiology of the illness. An even more recent article (Crandon 1981) is the sole representative ot the third category; articles exploring the epistemology ot susto. While her paper takes into account previous publications, it primarily focuses on the significantly different question: "why susto'?" Why is susto chosen as a diagnosis from the total array of ailment labels available to members of a culture, especially when many have similar or identical symptoms? Crandon further asks, "what is the socio-cultural meaning ot this choice?" Her answer is that the diagnosis, "susto." symbolizes the negotiation of and is a symbolic reflection ot socio-cultural identity, economic status, and the perception of self-worth in the community she studied.

Even given this broad treatment of the subject, three dimensions of susto appear to have been either partially or totally neglected in the previous studies. These dimensions both shed light on the controversies being addressed by the above authors, and provide an expanded framework within which those controversies can be better understood. First, a description ot the incidence and prevalance of susto as a part of the overall morbidity patterns of Latin American communities is missing. The closest anyone has come to such a quantitative treatment of the subject is in the work of Rubel and O'Neil (1979) in their analysis of the importance of susto within clinic populations. However, by the very nature of a clinic population, their data cannot be generalized to the wider 


\section{ETHNOLOGY}

community. Second, there are no descriptions of the range or the frequency of use of specific treatments for susto; again within a community based context Finally, no attempt has been made to empirically demonstrate whether susto is an isolated illness or is a part of a larger cluster of illnesses that are linked within a given ethnomedical system. Commonly, previous studies have simply lumped susto together with the other "culture bound syndromes" found in Latin American communities; ailments such as empacho (blocked intestines), mat de ojo (evil eye) or caida de moellera (fallen fontenelle). This lumping appears to be accomplished under the weak logic that these illness must have something in common since none of them fit scientific medical categories and all of them involve a belief in supernatural forces. The data presented in this paper provide evidence that there is a more logical and empirically sound method for clustering ailments within an ethnomedical treatment system.

\section{RESEARCH AREA AND METHODOLOGY}

The data for this article were collected in the Lower Rio Grande Valley of Texas. The Valley, as it is called by its residents, is the area bordering the Rio Grande river from Brownsville, at its mouth, upriver to Rio Grande City. Four Texas counties are included in the area; Starr, Hidalgo, Willacy, and Cameron. The approximately half-million people living in the area have an ethnic composition that is about 8c per cent Mexican American, 20 per cent Anglo American, and less than 5 per cent Black, Native American, or Asian. The two Mexican border towns of Matamoros and Reynosa, (Tamaulipas) are directly adjacent to the Valley and are very important to the overall cultural dynamics of the region.

The region, the people, and the major enthnomedical system of the area curanderismo, have been recently described in detail (Trotter and Chavira 1980, 1981). When these works are coupled with earlier descriptions or ethnographies (e.g., Bourke 1894, Rubel 1960, 1964, 1966; Madsen 1964; Romano 1960 1964, 1965), the lower Rio Grande Valley is shown to have a greater historical depth for the academic treatment of Latin American folk medicine, and especially for susto. than nearly any other area in the world. Thus, it seems unnecessary to reiterate here the information combined in those earlier works, and their bibliographies.

The study of susto reported in this paper grew out of a broader study of the home treatment of illnesses in the region. The author has established a computer based data bank of remedios caseros (home remedies) collected in the Valley. This data bank contains coded cases of the uses of home remedies sampled from the broad four-county region. Each case consists of a single home remedy, the illness it treats, its method of preparation, a case example of its use, and general demographic information about the informant (e.g., age, sex, ethnicity, occupation, country of birth, residence, languages spoken, etc.). Currently, over 4,000 cases have been collected. These cases come from members of the various ethnic groups found in the Valley as well as from a number of foreign nationals. For the purposes of this study, a sample of 1,223 cases was drawn from the total computerized archive. This sample contains all of the cases presented by informants who identified themselves as Mexican Americans. The sample includes information from 378 informants ranging in age from 16 to 82, but clustering most heavily in the 30 to 55 age rang-. While all sample informants were identified as Mexican Americans, 41.4 per cent of the cases were provided by informants born in Mexico; 58.6 per cent by informants born in the United States; 85.4 per cent of the cases were presented by female informants and 14.6 per cent by male informants.

The sample should be considered a convenience sample since it has thus far been economically impossible to minutely stratify the sample collection on the basis of small geographical units in the Valley. Nevertheless, a general inspection of the data indicates that the social and geographical characteristics of the informants match the overall conditions of the Valley. Furthermore, the data are consistent with both the ethnographic data previously collected in the Valley and with the incidence and prevalence of illnesses collected locally from more conventional sources of information (e.g., Public Health surveys).

Five hundred and ten separate botanical and nonbotanical remedies (including single ingredients and/or combinations of ingredients) were discovered in the sample. The ethnographic evidence indicates that the remedies, and the ailments they treat, should not be randomly distributed in the sample. A computer aided analysis (Trotter 1981 a) confirmed that there is a core group of 56 remedios (approximately 11 per cent of the total number of remedies) that account for over 52 per cent of the cases in the sample. It was further demonstrated that a core group of 60 ailments, out of a total of 198, accounts for more than 81 per cent of the cases in the sample. A subsequent analysis of the data, using the demographic variables collected for each case, demonstrated that it was possible to generate a profile of community based morbidity patterns of home treated illness (Trotter 1981b), and that these could be broken out into age and sex specific categories comparable to conventional morbidity studies. The following section presents a summary of the data from the sample of cases which is pertinent to susto and to a presentation of the three neglected dimensions of susto mentioned above.

THE PROMINENCE OF SUSTO AS A HEALTH PROBLEM

Since the informants in the sample were allowed to present whatever ailments and remedies that occurred to them without forced choices, each of the ailments can be considered a part of the overall morbidity problems treated in the home. Morbidity is defined as:

basically a departure from a state of physical or mental well being, resulting from disease or injury, of which the individual is aware. Awareness connotes a degree of measurable impact on the (National Center for Health Statistics 1964 ).

If it is assumed that the remedies most frequently recalled and presented by the informants in the sample represent the treatment of either the most commonly encountered ailments or the most important ailments treated in the home, then the frequencies presented in Table 2 can be considered as a comparative measure of the prominence of a particular ailment within the home treatment system (see Trotter $1981 \mathrm{~b}$ for detailed discussion of this premise). By the same logic, _both ailments and remedies which show up infrequently in the sample are of less importance to the community as a whole. These assumptions are supported by the condition that no new ailments were discovered in the sample beyond the time when approximately half of the cases had been coded. Thus, the total list of ailments appears to represent all or most of the illnesses treated in the home by Mexican Americans in the Valley, within the limits of any sampling error. New remedies continued to show up throughout the coding process, but only at the rate of one or two for each fifty cases coded, past the time that approximately two-thirds of the cases had been coded. Most of those discovered at that time were simply new combinations of previously coded herbs. The following table provides a comparison of the 30 most frequent ailments in the sample of cases.

Table i demonstrates one of the primary contentions of medical anthropology that culture has a significant effect on the morbidity patterns of a community. There are three ailments in the list that have no English language equivalents. These are nervios, susto, and empacho. Two of the three have been prominent in the anthropological discussions of Latin American ethnomedicine, while the third, »en<ios. has been neglected. Although nervios can be generally translated as 
anxiety or nervousness, there is current ethnographic and linguistic evidence from the Valley to support the idea that it, perhaps, should be considered as a culture bound syndrome separate from those other two conditions. Thus anthropologists have been correct in demanding that these ailments not be ignored, since they represent conditions that are a departure from a state of well being of which the individual afflicted is aware. At the same time, as stated elsewhere (Trotter 1981b) there has been a significant neglect of the physical aspects of health conditions in the anthropological treatment of Hispanic folk medicine in favor of descriptions and analyses of the culture bound syndromes. This article serves to redress that imbalance.

The data in table $\mathrm{i}$, and the lengthier tables of relative frequencies of the ailments presented elsewhere (Trotter 1981 a,1981b) have a twofold usefulness. The first is to compare the prominence of susto to all of the other ailments treated in the home. The second is to compare the prominence of all of the culture bound syndromes to one another. Although there are numerous descriptions of these

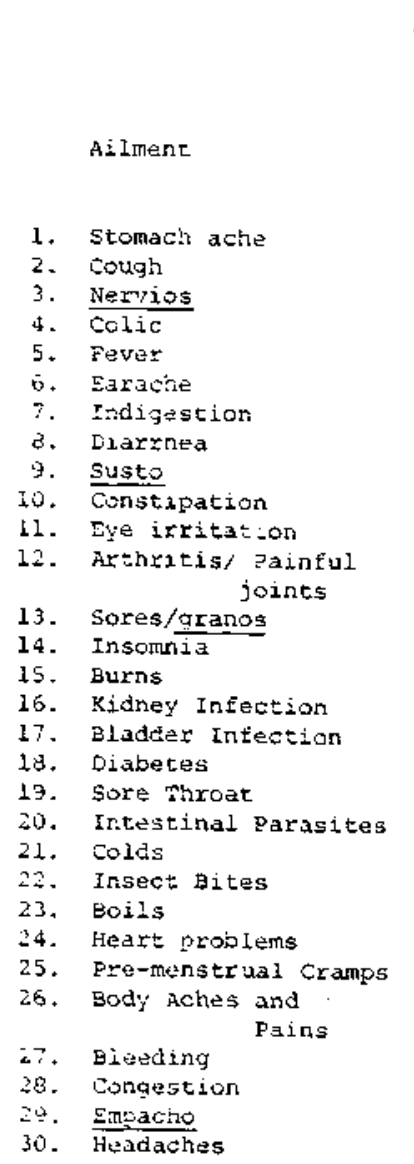

The Thirt: Most Cormon Ailments

in the sample of cases ${ }^{1}$

Numicer of cases Percentage of

Parcontage of Cummulatín

percentage

$\begin{array}{lrr}76 & 6.2 & 6.2 \\ 54 & 4.4 & 10.6 \\ 49 & 4.0 & 14.6 \\ 44 & 3.6 & 18.2 \\ 44 & 3.6 & 21.8 \\ 37 & 3.0 & 24.8 \\ 37 & 3.0 & 27.8 \\ 36 & 2.9 & 30.7 \\ 35 & 2.9 & 33.5 \\ 29 & 2.4 & 35.9 \\ 27 & 2.2 & 38.1 \\ & & \\ 26 & 2.1 & 40.2 \\ 24 & 2.0 & 42.2 \\ 23 & 1.9 & 44.1 \\ 20 & 1.6 & 45.7 \\ 20 & 1.6 & 47.3 \\ 19 & 1.6 & 49.9 \\ 19 & 1.0 & 51.4 \\ 19 & 1.6 & 53.0 \\ 18 & 1.5 & 54.5 \\ 15 & 1.2 & 53.7 \\ 15 & 1.2 & 54.9 \\ 14 & 1.1 & 56.0 \\ 14 & 1.1 & 57.1 \\ 14 & 1.1 & 58.2 \\ 13 & & \\ 13 & 1.1 & 59.3 \\ 22 & 1.1 & 60.3 \\ 12 & 1.0 & 61.3 \\ 12 & 1.0 & 62.3 \\ & 1.0 & 63.3\end{array}$

illnesses in Latin American communities, no one has quantitatively compared their frequency distributions within a community. Table 2 presents the frequencies of cases for the folk ailments most commonly identified in the ethnomedical literature as they occur in the sample of cases presented in this article. Nemos is not included, pending further research on it as a culture bound syndrome.

Together these tables present an interesting perspective on susto. It represents nearly 3 per cent of all of the cases in the sample, making it the ninth most prominent morbidity problem in home treated ailments. It is two-and-one-half times as frequent in this sample as malde ojo, caida de mollera. bad luck and ridding evil spirits combined. Susto is nearly three times as common as empacbo, the second most prominent of the house treated folk illnesses, yet empacho is far more common than the other ailments. ${ }^{2}$ Taken together, the six ailments represent 3 per cent of the 198 ailments in the sample and 5 per cent of the 1,223 cases. Similar samples from the regions described by other ethnographers will have to be analyzed before a comparison can be made of the relative importance of susto, and other ailments, in these communities.

\section{A COMMUNITY PERSPECTIVE ON THE TREATMENT OF SUSTO}

As stated above, there are many solid ethnographic descriptions of the treatment of susto (Rubel 1960, 1964; Madsen 1964; Trotter and Chavira 1981) in the Lower Rio Grande Valley. Based on those data, the general impression is that susto is a magico-religious ailment in which a startling, frightening, or shocking experience causes some level (from weak to total) of disengagement of the soul from harmonious syncopy with the body. This loss of syncopy then produces the physical symptoms described by Rubel (1964) which include insomnia, fatigue, irritability (jumping at sudden noises), anxiety and nervousness, lack of interest in personal hygiene and appearance, weakness, paleness, and in some cases nausea, vomiting, diarrhea, and/or fever. The most common treatment for the ailment depicted in the ethnographic literature is a magico-

Frequenties of FolK Ilinesses

in Saxple of cases

\begin{tabular}{|c|c|c|c|c|}
\hline Ailment & $\begin{array}{l}\text { Total } \\
\text { Casses }\end{array}$ & $\begin{array}{c}\text { Percent of } \\
\text { A11 Sample Cases }\end{array}$ & $\begin{array}{l}\text { Cumulative } \\
\text { Percentage }\end{array}$ & $\begin{array}{l}\text { Percent of } 61 \\
\text { Eolk ilinesses Cases }\end{array}$ \\
\hline susto & 35 & 2.9 & 2.9 & 37.4 \\
\hline etmeacho & 12 & 1.0 & 3.9 & 19.7 \\
\hline 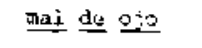 & 5 & 1). 4 & 4.3 & 8.2 \\
\hline $\begin{array}{l}\text { rid perzon of } \\
\text { ev il spirits }\end{array}$ & 5 & 0.2 & 4.7 & 8.2 \\
\hline$\Rightarrow 1$ minate & 3 & 0.1 & 4.7 & 4.9 \\
\hline caida de & 1 & 3.1 & 5.0 & $\begin{array}{r}1.6 \\
- \\
\end{array}$ \\
\hline & 51 & 5.0 & 5.0 & 100.0 \\
\hline
\end{tabular}


religious ritual called a barrida (sweeping) which uses physical objects and incantations to supernaturally remove the harm being caused by susto. This treatment fits the harmonious logical structure of a supernatural disease (often called psychosomatic in the literature) being treated by supernatural (i.e., psychosomatic) means. Unfortunately, the data collected in the survey do not match this neat logical package, as can be seen in Table 3.

By far the most common treatment of susto in this sample ( 40 per cent of the total cases of susto) is the administration of a tea made from Yerba aniz. (Pimpinella anisum L.; anise). A total of 61.7 per cent of the cases, excluding the one where the method of administration is unknown, were treated by teas, and an additional 5.9 per cent by ingesting either sugar or a mixture of vinegar, salt, and water. Only 32.4 per cent of the cases were treated by means of a barrida, and a mild case can be made that albacar (Ocimum basilicum L; (sweet basil) and cenizo (Leucophyllum texanium Beuth; purple sage) may have some biochemical effect as inhalents. These data, then, appear to support the statements of Gillen (1945, 1948), Bolton (1980), and Crandon (1981) that susto may have underlying physiological causes or correlations. On the other hand, these remedies may simply be effective in treating some of the symptoms of susto, rather than the ailment itself. A parallel case is found in the treatment of the common cold by scientific medicine, and this hypothesis is supported by the data in Table 4. Table

Table 3

Relative Frequencies of Susto Remedies ${ }^{3}$

and Their Method of Administration

Remedy

1. manzanilia

2. yerba niniz

3. yerba buena

4. hoja de naranjo

5. alkacar

6. $\underline{\text { cenixo }}$

7. toronjis

8. altamisa

9. epasote de zorrislo

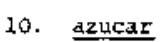

11. 51monillo

12. 미느

13. jerbant1lo

14. agua, vinaqre, sal

\begin{tabular}{c} 
No. of \\
Cases \\
2 \\
14 \\
1 \\
1 \\
4 \\
1 \\
1 \\
3 \\
1 \\
1 \\
1 \\
3 \\
1 \\
1 \\
\hline 35
\end{tabular}

\section{Percent of} Susto cases

5.7

40.0

2.9

2.9

11.4

2.9

2.9

8.6

2.9

2.9

2.9

8.6

2.3

2.9

100.0
Cumulative

Percent

5.7

45. 7

48.6

51.4

62.8

65.7

68.5

77.1

80.0

82,8

85.6

94.2

97.1

100.0

$\longrightarrow$
Mettiod of Administration

tea

tea

tea

tea

1 taa, 3 barridas

parrida

tea

barrida

barrida

tsp. w/out water

unknown

barrida

tea

mix and ingest

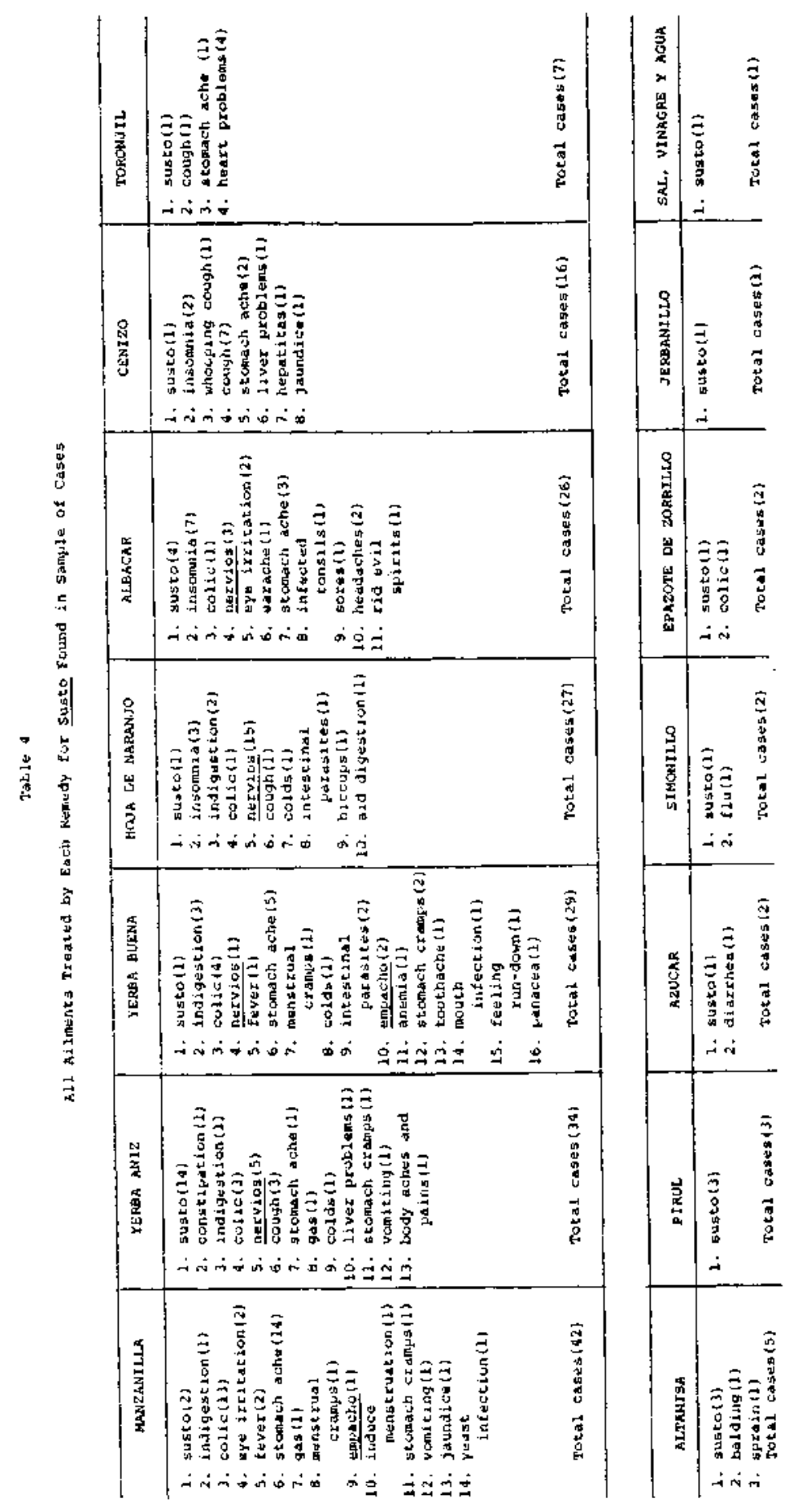


4 was created by using the computer to generate the frequency data on all of the ailments treated by each of the remedies caseros that are used to treat susto.

Even a casual inspection of Table 4 indicates that many of the remedies that treat susto also treat its symptoms, or treat problems that are both recognized as ailments in and of themselves, or as symptoms of susto when they occur in combination with other conditions. The extent of the clustering of ailments within the group of remedies that treat susto is presented in Table 5.

Out of a total of 42 ailments that have at least one treatment in common with susto. 17 (40.5 per cent) have at least two treatments in common and 7 (16.7 per cent) have three or more treatments in common. If the various stomach ailments (e.g., colic, indigestion, etc.) can be lumped under the general rubric "nausea," then nine out of the seventeen listed ailments are symptoms that accompany susto. A more conservative estimate would include only insomina, fever, vomiting, indigestion (adult susto symptoms), and colic (children's susto symptoms), lowering the percentage of susto symptoms in the above list from 52.9 per cent to 29.4 per cent. Two other symptoms of susto occur in the group of 24 ailments that have only one treatment in common with susto. These are diarrhea and feeling run down. A third ailment, anemia, would produce the symptoms of paleness and weakness associated with susto. Thus, within the total group of ailments that are treated by the remedies that treat susto, there appear to be a set that have a common symptomology with susto.

There also appears to be an additional set of ailments (e.g., eye irritation, sprained joints, hiccups, etc.) that are not symptomatically related to susto, but are linked to it through having one or more treatments in common. Following the logic presented earlier, this methodology of determining the treatments of one illness, then linking those treatments to the other illnesses that have these treatments in common, does appear to produce clusters of ailments that define common spheres of ethnomedical activity. At the same time, the level of variation within these clusters will necessitate considerable further analysis to determine the structural principles underlying these clusters, beyond the explanation of

Table 5

Ailments That Have Two or More Treatments in Common

With Susto

Ailment (No. of Treatments in Common)

$\begin{array}{ll}\text { 1. } & \text { Colic } \\ \text { 2. Stomach ache } & (6) \\ \text { 3. Nervios } & (4) \\ \text { 4. Indigestion } \\ \text { 5. Cough } \\ \text { 6. Colds } \\ \text { 7. Insomnia } \\ \text { 8. Fever }\end{array}$

(6)

(6)

(4)

(4)

(4)

(3)

(3)

(2)
10. Gas

11. Menstrual Cramps

12. Liver Problems

13. Jaundice

14. Intestinal Parasites (2)

15. Empacho

16. Stomach Cramps

17. Vomiting clustering due to the correspondence of symptoms among similarly treated ailments. It cannot be determined at this time what the ethnomedical relationship between susto and the nonsymptomatic ailments is, if any, beyond common treatments. However, that attempt, along with a statistical technique for describing the clustering effect, will be the subject of a subsequent article.

\section{SUMMARY AND CONCLUSIONS}

Three things are accomplished in this article. The relative position of susto is established within the overall morbidity patterns of a sample of cases of home treated illnesses within the Lower Rio Grande Valley of Texas. For the first time, the range and variation of treatment for the ailment are presented in a way that allows quantitative comparisons with other populations where susto exists within the community ethnomedical system. Finally, a preliminary descriptive method for clustering ailments that represent common spheres of activity within the home treatment of ailments in a community is presented.

This information provides a base to reassess the data and conclusions of earlier works on susto. The fact that over 60 per cent of the treatments for susto are ethnopharmacologicai, as opposed to magico-religious, lends some credence to the physical causation hypothesis for susto. The major current proponent for this position, Bolton (1980, 1981), develops the thesis that hypoglycemia causes the diagnosis of susto in Latin American populations, or, perhaps more accurately, that susto is the Latin American label for an equivalent disease labeled hypoglycemia in medical terminology. He bases this linkage of susto and hypoglycemia on a tripartate correlational analysis of the statistical relationships he discovered between people he empirically identified as having hypoglycemic tendencies, the presence or absences of high levels of hostility in individuals, and reported episodes of the presence or absence of previous or current susto diagnoses for his subjects. Unfortunately, while he carefully documents the correlations, there is one serious confounding problem in the logic behind his methodology. He bases the identification of an individual with hypoglycemic tendencies on a combination of "GTT (Glucose Tolerance Test) results and on their hypoglycemia symptom scores," (Bolton 1981:262). The latter scores were derived from a questionnaire designed to elicit the presence or absence of hypoglycemia symptoms. However, Bolton (1981:262) already demonstrated that "the symptoms usually described in the literature for susto patients and the most frequent complaints of hypoglycemia patients coincide." Therefore, if the hypoglycemia symptom scores form a significant part of the identification of someone with hypoglycemic tendencies, those persons should a priori have a very strong correlation with a past or present susto diagnosis, since the hypoglycemia symptom scores are also symptom scores for the hypoglycemia equivalent called "susto." In other words, Bolton has created a questionnaire that acts as a measure of susto symptoms (and, apparently, hypoglycemia symptoms) and has discovered that a high score on the instrument correlates very strongly with a past or present diagnosis of susto for that person; and he has found that persons who have a very low score on the susto symptom measure tend not to have been diagnosed as having susto. Stated this way, his results are not at all surprising, and in some ways they provide an interesting confirmation of some of the contentions set out by Fabrega (1970) on the specificity of folk illnesses in a population. Bolton's (1981) thesis fails, in its logical structure, to do more than show a strong correlation between susto symptoms and susto diagnosis. Starting with the same logic and methodology, it should be possible to produce similar correlations between any, and perhaps all, other diseases that share the major part of their symptomatology with both hypoglycemia and susto. such as those identified by Crandon (1981); i.e., vitamin B complex deficiencies, anemia, etc. In fact, those ailments become more 
plausible causal factors for susto in the Valley, given a second part of the logical structure in Bolton's hypoglycemia hypothesis. He (Bolton 1981:274) states that

there are many sources of hypoglycemia in Incawatana, e.g. hypoxic stress, dietary deficiencies, that makes so many individuals in Incawatana prone to hypoglycemia.

Embedded in this statement, since he equates hypoglycemia and susto, is the implication that it is these stressors which produce the high level of susto in the population, and that without them susto would be drastically reduced or disappear. Unfortunately, he does not provide a quantitative measure of the incidence of susto which can be compared to the one derived for the Lower Rio Grande Valley data. However, given that susto is the ninth most common home treated ailment in the Valley sample, it is interesting to note that the elevation of the Valley at its highest point is 30 meters above sea level (hypoxia is rare), that the coldest temperatures experienced are normally four to six days per year in the 50 degree fahrenheit range, and that several nutritional studies have uncovered consistent vitamin A and D deficiencies, but no serious malnutrition endemic to the Valley population. The disease load in the Valley is heavy for the United States, but not heavy in comparison to the area represented by Bolton's research. And a spot survey of local physicians indicated no abnormal levels of hypoglycemia compared to the United States as a whole. Therefore, these conditions appear to weaken the hypoglycemia thesis and would have to be addressed before the Bolton hypothesis could be extended to the Lower Rio Grande Valley.

The evidence presented in this article does not affect the social causation hypothesis for susto in the same way that it does the above thesis. Social stress is certainly present in more than sufficient quantity, given the social, economic, and cultural dynamics of the region. Susto is available to be manipulated as a way of imposing control over social situations and definitions, and there is a constant negotiation and symbolization of identity among various alternatives presented by a multicultural environment. At the same time, susto is normally described by the social causation authors as a magical condition. Several of the authors use the phrase "magical fright" in the titles of their articles, and most include the idea that the disease includes the condition of "soul loss" as one of the ethnomedical elements of the ailment. Yet only about one third of the treatments in the sample are ritual in nature: which puts the social causation theorists in the position of having to find ethnographic evidence of the logical construct that an herbal tea is better (or at least more commonly used) for restoring a soul into a syncopy with the body than is a supernatural ritual. Rather than following such a course, an alternative condition is suggested based on the quantitative data provided here combined with the author's ethnographic data.

The combined ethnographic and quantitative data suggest that there is a cluster of symptoms/ailments that informants tentatively label susto, or label as individual ailments which are also symptoms of susto. There is a relatively wide choice of treatments for the ailments, the most common of which are herbal teas. If the "problem" goes away after its treatment, then the original diagnosis is probably confirmed in the mind of the informant, along with the reinforcement of the efficacy of the treatment. If the "problem" persists, or additional symptom appear, other treatments may be attempted. Thus, the diagnosis is either reinforced, changed from susto symptom to susto, or changed away from susto. While there is no way of determining the treatment progression from the quantitative data, the ethnographic evidence suggests it may typically be from herbal to magical, and from treatment within the family context to treatment by curandero and/or physician. This contention is supported by the comments of one curandero interviewed by the author who was asked if he treated susto, and if so, how often. He replied that, most of the time, there was no need for him to do so, since "everyone knows how to treat it, your grandmother, your aunt, you comadre." Instead, he only treated sustopasado (advanced susto). This, he and other curanderos stated, is more dangerous and must be treated through ritual processes of the type normally only attempted by curanderos. When asked to distinguish between the alternate forms of susto and susto pasado, the criteria appeared to be that if it was successfully treated by teas and the simple barridas your family might give you, it wasn't susto pasado. and if it wasn't successfully treated that way, it was susto pasado. Left untreated, susto pasado is thought to be potentially fatal.

When the curandero's comments are juxtaposed with my data and with Crandon's contention (1981) that susto is different for adults and children, at least among the Aymara, an interesting supposition results. The overall variation in susto that is implicit in these three lines of evidence, suggests that susto is actually a complex set of closely related ailments, as opposed to a single ailment. This makes susto analogous to the complex illness we call cancer. Cancer, like susto, has competing theories of causation, including various pyschosomanc hypotheses; and significant strides in understanding cancer were made only after some of the individual variants of the ailment were isolated and were no longer masked by the total range of syntomology and variation within the disease complex as a whole. This analogy suggests that if susto is a set of related ailments, rather than a simple ailment, it will be understood, and all of the lines of evidence will converge only after it is treated to a more particulate, rather than an incorrectly directed global, analysis. This approach may make it possible to reconcile some of the competing theories of causation by testing them against a properly defined variant of the illness. Thus, instead of confirming or challenging the social hypothesis of previous authors, the data presented in this paper suggest that a re-evaluation of susto itself needs to be undertaken to examine the variability inherent in the ailment both within and between cultures. Only in this way are the competing theories of causation likely to be resolved.

\section{NOTES}

1. There are some minor differences in the rauk order of the ailments listed here and those listed in Trotter (ituSia, i98ib). These are caused by the discovery ot incorrectly coded cases when the coding of all cases was rechecked tor this data run.

2. This creates an intriguing question tor future research. Even though it is the second most common folk illness, it has by tar the least prominent position in the literature. Is this neglect because ampacho is the most physical of the folk ailments, and lacks the ritual elaboration common to the others?

3. The binomial designations for the remedies, where they are botanical, are as follows: Manzanilla (camomiie) Matncaru : hamomilla L.; Yerba amz (anise) Pimpinella anisttm L, Yerba Buena (mint) Mtntha spicata L.; lioja de Naranjo (orange leaves) Citrus auranttum L.: Albacar (sweet basil) Olimum basilicum L., Cenzo (purple sage) Leucophyllum texanum Beuth. Toronjil igentle balm) Schinus sflle L. L.; Altar (ta (mar): Simonillo (unknown) Epazote de Zorllo (skunk weed) Chtnopodtum grateo lens Las. Jerbamllo (unidentified plant); Sal, vinagre, agua (mixture ot salt, water, and vinegar).

\section{BIBLIOGRAPHY}

Bolton, R. 1980. Susto: The Myth of a Folk Illness. Paper presented at the "9th annual meeting ot the American Anthropological Association, Washington, D.C.

Bourke J. 1981. Susto, Hostility, and Hypoglycemia. Ethnology 19:261-276. American Folklore 7:119-146.

Crandon, L. ly Hr. A Historico-Ecological Model for Susto. Paper presented at the Both annual meetings ot the American Anthropological Association. December, Los Angeles.

Fabrega, H. Jr. 1970. On the Specificity of Folk Illness. Southwestern Journal of Anthropology. 26:305-314.

Grebe, M. E., and J. Segura. 1974. Psiguiatna Folklonca de Chile: Estudio Anthropologico de sus Enfermedades Vigentes. Aeta Psiguiatrica y Psicologica de America Latina 20:307-382. 


\section{ETHNOLOGY}

Gillen, J. 1945- Moche: A Peruvian Costal Community. Smithsonian Institution, Institute of Social Anthropology, Publication \#3, Washington

Goebel. O. i9 3- El Susto: A Descriptive Analysis. International Journal of Social Psychiatry

Kiev, A. 19:38-43. Curanderismo: Mexican American Folk Psychiatry. New York.

Logan, M. 19"9- Variations Regarding Susto Causality Among the Cakchiquel of Guatemala, Culture Medicine and Psychiatry 3:1-13-166.

ricans of South Texas. New York.

Nail, F. C., and J. Speilberg. 196". Social and Cultural Factors in the Response of Mexican Americans to Medical Treatment. Journal of Health and Social Behavior 8:299-308.

National Center tor Health Statistics. 1964. Health Survey Procedure: Concepts, Questionnaire Development and Definitions in the Health Interview Survey. Pubi

$\mathrm{O}^{\prime} \mathrm{Nell}$, C. W. 1975. An Investigation ot Reported "Freight" as a Factor in the Etiology of Susto

O'Nell, C. W.. and A. J. Rubei. 19^6. The Meaning of Susto (Magical Freight). Actas del Congreso International de Ainericanstas 3:343-349.

to Measure Social Stress in Three Mesoamerican Communities. Ethnology 19:111-12

O'Nell, C. W., and H. A. Selby. 1968. Sex Differences in the Incidence of Susto in two Zapotec Pueblos: An Analysis ot the Relationship between Sex Role Expectations and a Folk Illness. Ethnology -: oviO5.

1966-9-6nship in a Mexican American Community in Texas. American Anthropolgist 62:966-9-6.

1964. Don Pedrito Jaramillo. The Emergence of a Mexican American Folk Saint. Ph.D. 1965. Charismatic Medicine, Folk Healing, and Folk Sainthood. American Anthropologist 6 : i 151-1 173.

Rubel, A. J. 1960. Concepts ot Disease in a Mexican American Community in Texas. American Anthropologist 6.y.-95-814.

1964. The Epidemiology ot a Folk Illness: Susto in Hispanic America. Ethnology 3:268-

Rubei, A. J., and C. W. O'Nell. u; 9- Diricutades para Expresar al Medico los Trastornos que Aqueian al Paciente: La Entermedad del Susto, Domo Ejemplo. Boletin de la Oficin

Sal y Rosas. F. rgs8. El Mito del Jam o Susto de la Medicina indigena del Peru. Revista de la Sanuiad de Policia i8:i6 -2io.

Trotter. R. T. II. 1081 a. Folk Remedies as Indicators of Common Illnesses: Examples from the United States-Mexico Border. Journal of Ethnopharmacology $4^{\prime} \cdot 207-221$

i98ib. Remedios Caseros: Mexican American Home Remedies and Community Health Problems. Social Science and Medicine 1-58:107-1 14

Trotter, R. T., II. and J. A. Chavira. 1980. Curandensmo: An Ernie Theoretical Perspective of Mexican American Folk Medicine. Medical Anthropology 4:425-48

Uzzell, D., I9"4- Susto Revisited: Illness as Strategic Role. American Ethnologist 1:369-378. 